\title{
Industrial application of adaptive grid refinement - the case of FINETM/Marine
}

\author{
Jeroen Wackers \\ LHEEA Lab, CNRS EMR 6598 \\ Ecole Centrale de Nantes \\ 1 rue de la Noë, B.P. 92101, 44321 Nantes cedex 3, France \\ e-mail: jeroen.wackers@ec-nantes.fr
}

\begin{abstract}
Industrial application of mesh adaptation is gaining momentum, thanks to the growing awareness of its potential benefits [1] and the the ever increasing performance of the mesh adaptation software. However, the step to successful industry appliction puts requirements on mesh adaptation, which are different from those in an academic setting. This is a new challenge for research.

The FINETM/Marine hydrodynamic simulation suite [2] from NUMECA Int., which contains the flow solver ISIS-CFD created at Centrale Nantes, has featured an adaptive grid refinement capability since 2010. On the other hand, this technique has gained widespread use only in the last three years. As such, it is an interesting example to study what aspects of a mesh adaptation method dissuade and attract industry users. In this paper, I will provide my personal analysis of this question.
\end{abstract}

Naval architecture design offices and consultancy providers are often small enterprises, who do not have dedicated experts for CFD simulation. In bigger firms, the efficient production of large numbers of computations is essential. Thus, users often choose FINE/TM/Marine with its dedicated marine workflow, because the software makes it easy to produce accurate and reliable simulation results. This aspect is crucial for the user choice to apply mesh adaptation.

A first decisive factor is the ease with which the parameters for the adaptive refinement can be chosen. In the beginning years, clients often abandoned mesh adaptation because they did not know how to select the right settings. I will discuss how the user guidelines have been improved and simplified over the years and, by correlating this with the increase in active users, estimate the minimum required level of user-friendliness.

A second blocking point is the presence of bugs in the code, which dissuades users who are faced with tight production schedules. The improvement of the reliability was the main focus of mesh refinement development in FINETM/Marine around 2015. I will use data from a particular large-scale consultancy project to estimate the level of reliability that is needed for industry acceptation.

Finally, industry users are reluctant to change their simulation procedures, since they rely on a back catalogue of standardised simulations as a reference for new designs. This implies that an absence of dissuading factors is not enough; users only adopt mesh refinement if there is a significant positive reason to do so. In our case, widespread use was started by the emergence of new types of simulation that are impossible, or much too expensive, without mesh refinement. I will discuss the two most important ones: overset meshing and surface capturing for fast planing hulls.

For a researcher, it is important to accept that obtaining industry readiness is a slow process, which takes patience. For example, Hessian-based refinement which is becoming the standard in the aeronautical industry (see e.g. [3]) has been used in research with ISIS-CFD for 8 years, but has not yet been adopted by industry users. Still, the recent progress in several different industries is a sign that the introduction of adaptive meshing is worthwile; our patience can be rewarded.

\section{REFERENCES}

[1] J. Slotnick et al. CFD vision 2030 study: A path to revolutionary computational aerosciences. Technical Report NASA/CR-2014-218178, NASA (2014).

[2] https://www.numeca.be/product/fine-marine

[3] A. Balan et al. Verification of Anisotropic Mesh Adaptation for Turbulent Simulations over ONERA M6 Wing. AIAA Journal 58(4) pp. 1-16, (2019). 